\title{
Estenose Aórtica Grave em Pacientes Assintomáticos: o Dilema do Tratamento Clínico versus Cirúrgico
}

\author{
Severe Aortic Stenosis in Asymptomatic Patients: the Dilemma of Clinical versus Surgical Treatment \\ Marcelo Katz, Flávio Tarasoutchi, Max Grinberg \\ Instituto do Coração do Hospital das Clínicas da Faculdade de Medicina da Universidade de São Paulo - São Paulo, SP - Brasil
}

\section{Resumo}

A estenose valvar aórtica é cada vez mais prevalente concordante com o envelhecimento populacional. Por conseguinte, torna-se mais comum o atendimento de pacientes assintomáticos com estenose aórtica grave. Embora os pacientes com estenose aórtica grave sem sintomas façam parte de um mesmo grupo, são heterogêneos sob o ponto de vista clínico, laboratorial e ecocardiográfico. A abordagem desses pacientes traz à tona o dilema do tratamento clínico versus cirúrgico: submeter o paciente aos riscos da cirurgia ou mantê-lo em observação clínica sob o perigo do dano miocárdio irreversível ou mesmo da morte súbita? Sob esta perspectiva, baseando-se na literatura atual, este artigo fornece ferramentas que auxiliam na estratificação dos pacientes. A área valvar, grau de calcificação, velocidade de fluxo transvalvar aórtico, hipertrofia ventricular esquerda e teste de esforço alterado são os fatores que colocam os portadores de estenose aórtica grave assintomáticos em um grupo denominado de muito alto risco, em que a estratégia cirúrgica passa a ser considerada.

\section{Introdução}

A prevalência de estenose aórtica (EAo) é crescente, particularmente a de etiologia degenerativa, em grande parte relacionada ao envelhecimento populacional. O envelhecimento da população é um fenômeno mundial, e também acontece no Brasil. De acordo com estimativas do Instituto Brasileiro de Geografia e Estatística (IBGE), no ano de 2050, brasileiros com idade superior aos 75 anos de idade representarão $10 \%$ do total populacional' .

De acordo com Lindroos e cols. ${ }^{2}$, em um estudo ecocardiográfico de prevalência da EAo, aproximadamente 3\% da população acima de 75 anos de idade apresenta EAo grave de etiologia degenerativa ${ }^{2}$.

\section{Palavras-chave}

Estenose da valva aórtica, disfunção ventricular esquerda/ cirurgia, implante de prótese de valva, observação clínica.

\footnotetext{
Correspondência: Marcelo Katz •
}

Rua João Moura, 690/92 - Pinheiros - 05412-001 - São Paulo, SP - Brasil E-mail:mksp1975@gmail.com,mkatz@terra.com.br

Artigo recebido em 16/11/09; revisado recebido em 02/02/10; aceito em 24/02/10.
A importância epidemiológica da EAo pode ser estimada ao se projetar o cenário populacional brasileiro para o ano de 2050. O país terá 260.000.000 de habitantes, sendo que destes, aproximadamente $10 \%$, ou 26.000.000, serão pessoas acima de 75 anos de idade 1 . Considerando o estudo ecocardiográfico de Lindroos, teremos 800.000 pessoas acima de 75 anos de idade com EAo grave degenerativa. Somando estes pacientes àqueles portadores de EAo das demais faixas etárias e etiologias, poderemos considerar essa doença valvar como uma questão de saúde pública, aumentando ainda mais o interesse em seu estudo.

Além do aspecto epidemiológico, outra questão que se levanta em relação à EAo é a abordagem dos pacientes com EAo grave assintomáticos. Entende-se que o paciente assintomático seja aquele que não apresente os sintomas clássicos da EAo: dispneia, angina e síncope, independente da faixa etária e/ ou etiologia. O paciente com EAo grave, sem sintomas, tem sido tema de muito debate entre clínicos e cardiologistas nos últimos anos. De acordo com o paradigma anterior, baseado em observações da década de 60 e 70, os portadores de EAo grave sem sintomas poderiam ser observados clinicamente, desde que não apresentassem disfunção ventricular sistólica, uma vez que a curva de sobrevida desse grupo seria semelhante à da população em geral ${ }^{3}$.

Entretanto, o conceito de "benignidade" da EAo grave sem sintomas tem sido refutado nos últimos anos, basicamente apoiado em duas premissas:

1. Nem sempre o paciente assintomático está realmente livre de sintomas, muitas vezes procuram limitar progressivamente suas atividades, mascarando sintomas (principalmente idosos); são na realidade "pseudoassintomáticos", e, desta forma, tem pior prognóstico.

2. Os pacientes com estenose aórtica grave assintomáticos não são idênticos, ou seja, por mais que não tenham sintomas e que não apresentem disfunção ventricular esquerda, existem outras variáveis que podem aumentar ou diminuir risco destes pacientes, tornando-os, embora parte de um mesmo grupo, heterogêneos.

O objetivo deste artigo, portanto, é discutir, à luz das evidências atuais, a melhor abordagem do paciente com estenose aórtica grave assintomático, fornecendo ferramentas que auxiliem na decisão de mantê-lo em observação clínica ou submetê-lo ao tratamento cirúrgico.

\section{Estenose aórtica: aspectos relevantes}

A EAo é uma doença valvar que acomete a valva aórtica, 
e que se caracteriza pela obstrução à passagem do fluxo sanguíneo da via de saída do ventrículo esquerdo para a aorta. Tem como principais etiologias a doença reumática, degenerativa, também denominada aterosclerótica, e congênita ${ }^{4}$.

Qualquer que seja a causa da EAo, a via final é o processo de calcificação, e redução progressiva do orifício valvar. Na evolução da EAo, esta redução progressiva da área valvar determina hipertrofia ventricular esquerda (HVE), inicialmente com manutenção da função sistólica. A HVE permite que o paciente permaneça assintomático por um período variável de tempo. Com o passar do tempo ocorre desequilíbrio entre os compartimentos muscular, intersticial e vascular, resultando em isquemia e dano miocárdico. Há progressiva disfunção ventricular, inicialmente diastólica; na fase final observa-se disfunção ventricular sistólica.

$\mathrm{Na}$ história natural da EAo, o advento de sintomas (dispneia, angina e síncope induzida pelo esforço) é um marcador de gravidade, com implicações drásticas na curva de sobrevida destes pacientes ${ }^{3,5}$.

O diagnóstico da EAo baseia-se na anamnese, exame físico, e avaliação complementar. A anamnese deve ser criteriosa objetivando-se estabelecer a real capacidade funcional do paciente e identificar a presença de sintomas relacionados à estenose aórtica. É muito comum pacientes limitarem suas atividades com o advento dos sintomas ${ }^{6}$.

$\mathrm{Na}$ avaliação complementar, destaca-se o papel da ecocardiografia com Doppler (ECO $)^{7,8}$. O ECO permite a confirmação diagnóstica e a estratificação de gravidade da EAo, que pode ser leve, moderada ou grave. De acordo com a American Heart Association?, a EAo grave pode ser definida como aquela com área valvar menor que $1,0 \mathrm{~cm}^{2}$, gradiente médio transvalvar aórtico maior ou igual a $40 \mathrm{mmHg}$, e/ou velocidade de jato transvalvar aórtico no pico da sístole maior que $4 \mathrm{~m} / \mathrm{s}$. Uma subclassificação da EAo em EAo muito grave tem sido sugerida 9,10 . Seria aquela com área valvar aórtica menor ou igual a $0,7 \mathrm{~cm}^{2}$ e/ou área valvar indexada menor ou igual a $0,4 \mathrm{~cm}^{2} / \mathrm{m}^{2}$.

O tratamento definitivo da EAo grave, quando indicado, é cirúrgico, sendo que a o tratamento padrão, até o presente momento, é a troca da valva aórtica por uma prótese, que pode ser biológica ou metálica. As principais indicações para o tratamento cirúrgico são ${ }^{7,8}$ :

- EAo grave em pacientes sintomáticos (dispneia, angina e síncope)

- $\quad$ EAo grave em pacientes que serão submetidos à cirurgia de revascularização miocárdica

- EAo grave em pacientes que serão submetidos à cirurgia em aorta ou outras valvas

- EAo grave em pacientes com disfunção sistólica ventricular

Mais recentemente, tem sido utilizada à técnica de implante percutâneo da valva aórtica ${ }^{11,12}$. Trata-se de procedimento realizado por via arterial femoral, com colocação de prótese biológica aórtica ancorada em uma estrutura aramada que lembra um "grande stent", realizada em centros com experiência neste procedimento, sendo que ainda não substitui o procedimento cirúrgico convencional; reservada a casos selecionados em que há alto risco cirúrgico.

\section{Estenose aórtica grave em pacientes assintomáticos: o grande dilema}

A abordagem dos pacientes com EAo grave distingue inicialmente 3 grupos:

- Pacientes com EAo grave sintomáticos e/ou com disfunção sistólica do ventrículo esquerdo. Estes pacientes têm indicação formal ao tratamento cirúrgico, e desde que não haja fatores/comorbidades que contra-indiquem a cirurgia, esta deve ser considerada como tratamento de escolha.

- Pacientes com EAo grave sintomáticos e/ou com disfunção sistólica do ventrículo esquerdo, mas com comorbidades que contra-indiquem tratamento cirúrgico, ou ainda que recusem cirurgia. Nestes casos o tratamento será paliativo, embora haja perspectiva futura da possibilidade alternativa de tratamento com implante percutâneo de prótese valvar aórtica ${ }^{11,12}$.

- Finalmente, o terceiro grupo, composto por pacientes com EAo grave, assintomáticos, com função ventricular preservada, objeto maior deste artigo. Como destacamos, embora façam parte do mesmo grupo, são heterogêneos. A conduta, portanto será individualizada.

A decisão terapêutica para o paciente com EAo grave, assintomático, traz à tona o dilema: mantê-lo em observação clínica, ou operá-lo profilaticamente.

A estratégia de operar profilaticamente todos os pacientes com EAo grave assintomáticos não é viável. Imaginando que a "cirurgia profilática" fosse a rotina, estaríamos expondo 100\% dos pacientes com EAo grave assintomáticos a um risco de $3 \%$ a $4 \%$ relacionado ao procedimento cirúrgico, somado a um risco de $1 \%$ ao ano relacionado à presença de prótese valvar, beneficiando aproximadamente $1 \%$ desta população que teria o risco de morte súbita por ano $\mathrm{a}^{5,13-15}$.

Por outro lado, manter todos os pacientes desse grupo em observação clínica pode não ser seguro. Na realidade, estudos observacionais mais recentes relacionados à história natural da EAo grave em assintomáticos, mostram que este grupo não é tão "benigno" como se imaginava em décadas anteriores ${ }^{16-21}$.

Em 1997, Otto e cols. ${ }^{18}$ apresentam um estudo em que 123 pacientes com EAo grave assintomáticos foram acompanhados prospectivamente por 2,5 $\pm 1,4$ anos. Nesse estudo a probabilidade de sobrevida livre de sintomas para os pacientes assintomáticos foi de $93 \%$ no primeiro ano, $62 \%$ no terceiro ano e $26 \%$ no quinto ano. Através de análise multivariada, a velocidade de jato transvalvar aórtico, o aumento da velocidade de jato transvalvar aórtico e a mudança do status funcional foram preditores independentes de óbito ou necessidade de cirurgia. A probabilidade do paciente permanecer vivo, sem necessidade de cirurgia aos 2 anos de seguimento, foi de apenas $21 \%$ para os que apresentavam na inclusão do estudo velocidade de jato transvalvar aórtico maior que $4 \mathrm{~m} / \mathrm{s}$. 
Em 2000, Rosenhek e cols. ${ }^{21}$ publicam o resultado de um estudo prospectivo em que 126 pacientes portadores de EAo grave assintomáticos foram seguidos por $22 \pm 18$ meses. Ao final do acompanhamento, a probabilidade de sobrevida calculada para os pacientes assintomáticos foi de 93\% no primeiro ano, $91 \%$ no segundo ano e $87 \%$ no quarto ano. Através de análise multivariada, o grau de calcificação valvar foi preditor independente de eventos combinados que incluíram desenvolvimento de sintomas e/ou óbito. A probabilidade de sobrevida livre de sintomas para os pacientes assintomáticos que apresentavam calcificação valvar aórtica moderada ou intensa foi de $60 \%$ no primeiro ano, $47 \%$ no segundo ano e apenas 20\% no quarto ano.

Em 2001, Amato e cols. ${ }^{16}$ apresentam o resultado de um estudo prospectivo que acompanhou 66 pacientes com EAo grave assintomáticos. Todos os pacientes foram submetidos a teste de esforço em esteira, com o objetivo de estratificá-los. Houve quatro casos de morte súbita durante o acompanhamento, sendo que nestes, o teste de esforço havia sido positivo e a área valvar aórtica era menor ou igual a $0,6 \mathrm{~cm}^{2}$.

Em 2004, Bergler-Klein e cols. ${ }^{22}$ estudaram o BNP e o NTproBNP como marcadores prognósticos em pacientes com estenose aórtica. O foco era na análise da sobrevida livre de sintomas em assintomáticos. O valor de BNP menor que 130 pg/ml e de NT-proBNP menor que 80 pmol/l (678 pg/ $\mathrm{ml}$ ) foram preditores de sobrevida livre de sintomas por um período de 6 a 9 meses $^{22}$.

Em 2005, o grupo de Pellikka e cols. ${ }^{20}$ apresenta o resultado de um grande estudo em que 622 pacientes assintomáticos com EAo grave foram acompanhados prospectivamente por 5 anos. Nesse estudo, a probabilidade de pacientes não operados permanecerem livres de sintomas foi de apenas 33\% em 5 anos, enquanto que a probabilidade de sobrevida sem cirurgia foi de $25 \%$ em 5 anos. O risco de morte súbita, não precedida por sintomas, foi de aproximadamente $1 \%$ ao ano. Nesse trabalho, a partir de 2 anos de seguimento, o paciente assintomático passou a ter prognóstico pior do que a da população em geral, mesmo sem apresentar sintomas. Idade, insuficiência renal crônica e velocidade de jato transvalvar aórtico foram preditores de mortalidade ${ }^{20}$.

Mais recentemente, o grupo do Dr. Jean-Luc Monin e cols. ${ }^{17}$ conduziu um estudo que envolveu 107 pacientes com EAo grave assintomáticos. O objetivo era desenvolver um escore de risco que pudesse ser aplicado, e que fosse capaz de predizer a chance destes pacientes apresentarem eventos adversos ao longo do tempo. Os pacientes foram seguidos por 24 meses e óbito ou necessidade de cirurgia foram computados como evento adverso. Os preditores independentes de eventos adversos encontrados neste estudo foram utilizados para a construção do escore, que em seguida foi aplicado em uma segunda população de 107 pacientes com EAo grave, assintomáticos, com o intuito de validar o escore. As variáveis preditoras de desfecho foram sexo feminino, velocidade de jato transvalvar aórtico no pico da sístole, e o valor inicial do BNP. Uma formula foi construída para cálculo do escore:
Valor do escore $=[$ velocidade do jato no pico $(\mathrm{m} / \mathrm{s})$ $x 2]+[($ logaritmo natural do BNP) $x 1,5]+1,5$ (se sexo feminino)

Os valores obtidos para o escore foram agrupados em quartis: Q1 12,9; Q2 14,6; Q3 16,2 e Q4 19,7. A probabilidade de sobrevida livre de eventos em 20 meses foi de $80 \%$ nos pacientes do primeiro quartil e apenas $7 \%$ entre os pacientes do último quartil. Embora com resultados robustos, a aplicação sistemática de escore de risco, proposta pelo grupo do Dr. Monin ${ }^{17}$, necessita de validação para tornar-se rotina ${ }^{23}$.

Se por um lado, os estudos prospectivos que avaliaram pacientes com EAo grave, assintomáticos, mostram que o risco de morte súbita nesses pacientes é em torno de $1 \%$ ao ano, também revelam que o grupo é realmente heterogêneo, e que parâmetros clínicos, laboratoriais e ecocardiográficos determinam maior ou menor risco para estes pacientes.

O paciente assintomático além de ter o risco de morte súbita, tem também o risco de dano irreversível ao miocárdio em casos em que a cirurgia é postergada ${ }^{23}$. Esses riscos devem ser levados em conta quando da escolha da estratégia conservadora ou cirúrgica.

O que se tem buscado, portanto, é a estratificação mais refinada desse grupo de pacientes, com o objetivo de identificar, dentre os pacientes com EAo grave assintomáticos, aqueles de maior risco, e que, portanto, se beneficiam da cirurgia. O objetivo é evitar justamente a morte súbita e/ou o dano irreversível ao miocárdio. Neste propósito, e baseado em diretrizes e estudos observacionais mais recentes, os principais fatores de risco seriam ${ }^{7,8,10}$ :

- $\quad$ EAo muito grave (área valvar $\leq 0,7 \mathrm{~cm}^{2}$ ou área valvar indexada $\leq 0,4 \mathrm{~cm}^{2} / \mathrm{m}^{2}$

- Incremento acelerado de gravidade da EAo (definido como aumento na velocidade de jato transvalvar aórtico maior que 0,30 m/s ao ano)

- Calcificação valvar aórtica

- Presença de isquemia documentada/ doença arterial coronariana

- Disfunção sistólica ventricular (quando presente indica cirurgia)

- Dilatação ventricular esquerda com perda de função sistólica ("afterload mismatch")

- Hipertrofia ventricular esquerda excessiva ou rapidamente progressiva (12 a $14 \mathrm{~mm}$ em mulheres e 14 a $16 \mathrm{~mm}$ em homens)

- Idade (acima de 60 anos de idade)

- Outras comorbidade não cardíacas

\section{A resposta para o dilema: conduta individualizada}

Como exposto, embora pacientes com EAo grave assintomáticos façam parte de um mesmo grupo, na realidade são heterogêneos sob o ponto de vista clínico, laboratorial e ecocardiográfico. Como consequência, também o prognóstico 
torna-se dependente destas particularidades. Portanto, a conduta para estes pacientes deve ser individualizada. Em um extremo, para os pacientes de baixo risco, a conduta é conservadora, expectante. No outro extremo, para os pacientes de alto risco, a conduta é cirúrgica, com troca valvar aórtica.

Individualizar risco em pacientes assintomáticos também é a sugestão das principais diretrizes. De acordo com o consenso europeu, pacientes com EAo grave assintomáticos, com função ventricular preservada, que apresentem calcificação valvar exuberante, rápido aumento da velocidade de jato transvalvar aórtico, ou ainda teste de esforço alterado seriam candidatos à cirurgia, assim como aqueles que serão submetidos a outra cirurgia cardíaca concomitante, por exemplo revascularização miocárdica associada ${ }^{8}$. O consenso americano indica cirurgia para os pacientes que apesar de assintomáticos, apresentem calcificação valvar exuberante ou rápido aumento da velocidade de jato transvalvar aórtico ${ }^{7}$. A experiência nacional destaca a importância do teste de esforço na estratificação destes pacientes ${ }^{16}$.

Baseados no que foi exposto, propomos a seguinte abordagem para o paciente assintomático com EAo grave:

\section{Confirmação do diagnóstico de EAo grave}

O exame físico, complementado pela ecocardiografia permite a confirmação da EAo e a avaliação de sua gravidade. De acordo com os parâmetro ecocardiográficos, EAo grave pode ser definida como aquela que apresenta gradiente médio de pressão transvalvar aórtico maior que $40 \mathrm{mmHg}$, área valvar aórtica menor que $1 \mathrm{~cm}^{2} \mathrm{e} / \mathrm{ou}$ velocidade de jato transvalvar aórtico no pico da sístole maior que $4 \mathrm{~m} / \mathrm{s}$. Sugerese que EAo muito grave seja aquela em que a área valvar é menor que $0,7 \mathrm{~cm}^{2}$ ou a área valvar indexada é menor que $0,4 \mathrm{~cm}^{2}$. Quando houver dúvidas acerca da gravidade da EAo, uma boa alternativa é a avaliação hemodinâmica, com realização de manometria e detecção de gradiente de pressão transvalvar aórtico.

\section{Elucidação do real status funcional. Será que o paciente é realmente assintomático ou esta autolimitado?}

A anamnese destes pacientes deve ser detalhada e exaustiva, focada nas atividades cotidianas dos pacientes. É muito comum um paciente que caminhava sem problemas, agora, mais limitado, restringir-se nas tarefas domésticas, e mesmo assim dizer que se sente bem, quando na verdade deixou de executar o nível de esforço de outrora.

Uma boa alternativa complementar, na tentativa de elucidar a questão do status funcional, seria submeter esses pacientes a uma avaliação funcional, como por exemplo, o teste ergométrico ${ }^{24}$. O teste pode fornecer dados importantes e selecionar pacientes ditos pseudoassintomáticos. O teste também auxilia nos casos de síncope isolada (sem angina ou dispneia). A síncope isolada, como única manifestação clínica da EAo, é pouco comum ${ }^{25,26}$. Nesses casos, a presença de síncope durante o teste define a associação sintoma-esforço, indicando a cirurgia. Ressalte-se, ainda, que o teste de esforço pode inclusive ser associado à ecocardiografia ${ }^{27}$. A ecocardiografia de esforço é outro método de estratificação funcional destes pacientes.

Embora o teste de esforço seja pouco realizado na prática clínica $^{28}$, em metanálise recente ${ }^{29}$, que analisou o papel do teste ergométrico na avaliação de pacientes com EAo grave assintomáticos, os autores destacam que o teste é seguro e eficaz em identificar pacientes com alto risco para eventos cardíacos adversos e morte súbita. O teste pode ser utilizado para estratificação de risco e para definição do melhor momento de operar os pacientes.

O teste de esforço ganha cada vez mais espaço, e enquanto sua recomendação é classe llb no consenso americano de valvopatias $^{7}$ de 2006, a sociedade europeia de cardiologia em 2007 o coloca como benéfico e efetivo, incluindo o teste no algoritmo de tomada de decisão para os pacientes com EAo grave assintomáticos ${ }^{8}$.

\section{Avaliação da função ventricular esquerda}

A ecocardiografia permite a avaliação da função ventricular sistólica ${ }^{30}$. Nos pacientes com EAo grave, mesmo que assintomáticos, se houver disfunção ventricular sistólica, o tratamento cirúrgico deve ser considerado.

\section{Definição de conduta clínica ou cirúrgica para os pacientes com EAo grave, assintomáticos, com função ventricular preservada}

Nestes pacientes, a conduta deve ser individualizada ${ }^{10,31}$. Alguns parâmetros podem colocar o paciente em muito alto risco, e entre os fatores apresentados anteriormente, aqueles que definem muito alto risco são:

- Teste de esforço positivo (com sintomas evidentes ou hipotensão ao esforço).

- Área valvar aórtica menor ou igual a 0,7 $\mathrm{cm}^{2}$ ou área valvar indexada menor ou igual a $0,4 \mathrm{~cm}^{2} /$ $\mathrm{m}^{2}$. A indexação da área pela superfície corpórea é importante pelas diferenças antropométricas nos diversos pacientes.

- Velocidade de jato transvalvar aórtico rapidamente progressiva, com taxa de aumento anual maior que $0,30 \mathrm{~m} / \mathrm{s}$ ao ano.

- Calcificação valvar aórtica moderada a intensa se associada com rápido aumento da velocidade de fluxo transvalvar aórtico.

- Hipertrofia ventricular excessiva, outro marcador de gravidade, especialmente se somada à área valvar reduzida. A hipertrofia ventricular por um lado permite a manutenção do débito cardíaco frente à sobrecarga pressórica imposta pela EAo. Por outro lado, altera a função diastólica, diminui perfusão coronariana e está relacionada com aumento de mortalidade ${ }^{32}$.

Sugerimos, baseados na literatura atual, que pacientes assintomáticos com estenose aórtica grave, que apresentem os fatores de muito alto risco sejam candidatos ao tratamento cirúrgico. O risco cirúrgico é relativamente baixo comparado ao risco de rápido desenvolvimento de sintomas e morte súbita específico para esse grupo de muito alto risco. Embora ainda não haja estudo randomizado que aborde conduta clínica versus cirúrgica nesses pacientes, é cada vez mais aceita a 
estratégia de não retardar o tratamento cirúrgico, isto porque o dano miocárdio pode ser irreversível, os sintomas podem se desenvolver rapidamente sem a correta percepção do paciente, e o risco de morte súbita eleva-se drasticamente.

Uma consideração especial deve ser dada à população de idosos. Quando analisamos consensos e diretrizes, não há referência à idade-limite que contraindique o tratamento cirúrgico. Na realidade, o que os estudos revelam através de séries de casos, é que mesmo no idoso, o tratamento cirúrgico, quando indicado, é melhor do que conduta expectante ${ }^{33}$. Em se tratando do paciente idoso, deveremos buscar os fatores de muito alto risco, mas sempre levando em consideração a equação: expectativa de vida x qualidade de vida x risco do tratamento cirúrgico, para que a melhor decisão terapêutica seja tomada.

\section{Exemplos de situações clínicas}

Exemplificaremos o que foi discutido neste artigo através de três situações clínicas:

\section{Situação 1}

Homem de 53 anos, executivo, hipertenso e dislipidêmico, assintomático no dia-a-dia, joga futebol de campo com os amigos 2 vezes por semana. Em consulta com o cardiologista, detectado sopro sugestivo de EAo. Ecocardiograma revela: câmaras cardíacas com dimensões normais, espessura do septo e da parede posterior de $11 \mathrm{~mm}$, função biventricular normal, valva aórtica calcificada, com estenose aórtica grave, gradiente médio de pressão transvalvar aórtico de $45 \mathrm{mmHg}$, velocidade de jato transvalvar aórtico de 4,1 m/s, área valvar aórtica de $1 \mathrm{~cm}^{2}$. Demais valvas sem alterações. BNP de 70 $\mathrm{pg} / \mathrm{ml}$. O cardiologista orienta evitar o futebol, indica um teste de esforço, que é normal, e repete o ecocardiograma em 6 meses, cujo resultado é idêntico ao exame inicial. A conduta indicada é expectante, conservadora. Indicado retornos semestrais ou mais precoces se aparecimento de sintomas.

\section{Situação 2}

Homem de 75 anos, aposentado, assintomático no dia-adia, caminha regularmente. Em consulta com o cardiologista é detectado sopro sugestivo de EAo. O ecocardiograma revela: câmaras cardíacas de dimensões normais, espessura do septo e da parede posterior de $12 \mathrm{~mm}$, função biventricular normal, valva aórtica calcificada, com estenose aórtica grave, gradiente médio de pressão transvalvar aórtico de $45 \mathrm{mmHg}$, velocidade de jato transvalvar aórtico de 4,2 m/s, área valvar aórtica de $0,9 \mathrm{~cm}^{2}$. Demais valvas sem alterações. BNP de $100 \mathrm{pg} / \mathrm{ml}$. O cardiologista indica um teste de esforço, que é normal. A conduta inicial é expectante, com retorno em 6 meses. No retorno (6 meses) o paciente permanece assintomático, e novo ecocardiograma revela espessura do septo e da parede posterior de $14 \mathrm{~mm}$, função biventricular normal, valva aórtica calcificada, com estenose aórtica grave, gradiente médio de pressão transalvar aórtico de $58 \mathrm{mmHg}$, velocidade de jato transvalvar aórtico de 4,9 m/s, área valvar aórtica de 0,7cm². Observa-se rápida evolução da EAo em 6 meses (aumento da espessura de septo e parede, diminuição de área valvar, aumento de gradiente e velocidade de jato). O paciente é classificado como de muito alto risco pela rápida evolução da estenose aórtica e conduta indicada é cirúrgica.

\section{Situação 3}

Mulher de 62 anos, sem doenças diagnosticadas, assintomática no dia-a-dia, aposentada. A filha notou que a mãe tem saído menos à rua, e suas atividades são cada vez mais domésticas. O cardiologista detectou sopro sugestivo de EAo. Ecocardiograma revela: câmaras cardíacas com dimensões normais, espessura do septo e da parede posterior de $13 \mathrm{~mm}$, função biventricular normal, valva aórtica calcificada, com estenose aórtica grave, gradiente médio de pressão transvalvar aórtico de $58 \mathrm{mmHg}$, velocidade de jato transvalvar aórtico de 4,8 m/s e área valvar de 0,8 $\mathrm{cm}^{2}$. Demais valvas sem alterações. BNP de 200 pg/ml. O cardiologista indica um teste de esforço para avaliação do real status funcional da paciente: no segundo minuto do teste a paciente apresenta hipotensão com sensação de pré-sincope, e o teste é interrompido, com recuperação total da paciente. O cardiologista interpreta o caso como de muito alto risco, julga que provavelmente a paciente se autolimitava, e indica a cirurgia de troca valvar aórtica.

\section{Conclusão}

Os pacientes com EAo grave assintomáticos fazem parte de um grupo heterogêneo do ponto de vista clínico, laboratorial e ecocardiográfico. Destaca-se dentro deste grupo, uma parcela de pacientes classificados como de muito alto risco. Os critérios de muito alto risco são: teste de esforço alterado, área valvar aórtica menor ou igual a $0,7 \mathrm{~cm}^{2}$ ou área valvar indexada menor ou igual a $0,4 \mathrm{~cm}^{2} / \mathrm{m}^{2}$, velocidade de jato transvalvar aórtico rapidamente progressiva, calcificação valvar aórtica moderada a intensa, hipertrofia ventricular excessiva. Para os pacientes de muito alto risco a estratégia cirúrgica deve ser considerada frente à conduta conservadora.

\section{Potencial Conflito de Interesses}

Declaro não haver conflito de interesses pertinentes.

\section{Fontes de Financiamento}

O presente estudo não teve fontes de financiamento externas.

\section{Vinculação Acadêmica}

Não há vinculação deste estudo a programas de pósgraduação. 
Katz e cols.

EAo grave sem sintomas: conduta clínica $x$ cirúrgica

\section{Artigo de Revisão}

\section{Referências}

1. Instituto Brasileiro de Geografia e Estatística (IBGE).2008. [Acesso em 2009 out 15]. Disponível em http://www.ibge.gov.br/home/estatistica/populacao/ projecao_da_populacao/piramide/piramide.shtm

2. Lindroos M, Kupari M, Heikkila J, Tilvis R. Prevalence of aortic valve abnormalities in the elderly: an echocardiographic study of a random population sample. J Am Coll Cardiol. 1993; 21 (5): 1220-5.

3. Ross J Jr, Braunwald E. Aortic stenosis. Circulation. 1968; 38 (1 Suppl): 61-7.

4. Passik CS, Ackermann DM, Pluth JR, Edwards WD. Temporal changes in the causes of aortic stenosis: a surgical pathologic study of 646 cases. Mayo Clin Proc. 1987; 62 (2): 119-23.

5. Carabello BA. Evaluation and management of patients with aortic stenosis. Circulation. 2002; 105 (15): 1746-50.

6. Cheitlin MD. Asymptomatic adult patients with aortic stenosis: should they ever have aortic valve replacement? Am Heart Hosp J. 2005; 3 (4): 243-6.

7. Bonow RO, Carabello BA, Kanu C, de Leon AC Jr, Faxon DP, Freed MD, et al. ACC/AHA 2006 guidelines for the management of patients with valvular heart disease: a report of the American College of Cardiology/American Heart Association Task Force on Practice Guidelines (writing committee to revise the 1998 Guidelines for the Management of Patients With Valvular Heart Disease): developed in collaboration with the Society of Cardiovascular Anesthesiologists: endorsed by the Society for Cardiovascular Angiography and Interventions and the Society of Thoracic Surgeons. Circulation. 2006; 114 (5):e84-231.

8. Vahanian A, Baumgartner H, Bax J, Butchart E, Dion R, Filippatos G, et al. Guidelines on the management of valvular heart disease: the Task Force on the Management of Valvular Heart Disease of the European Society of Cardiology. Eur Heart J. 2007; 28 (2): 230-68.

9. Rahimtoola SH. Perspective on valvular heart disease: an update. J Am Coll Cardiol. 1989; 14 (1): 1-23.

10. Rahimtoola SH. Valvular heart disease: a perspective on the asymptomatic patient with severe valvular aortic stenosis. Eur Heart J. 2008; 29 (14): 1783-90.

11. Vahanian A, Alfieri O, Al-Attar N, Antunes M, Bax J, Cormier B, et al. Transcatheter valve implantation for patients with aortic stenosis: a position statement from the European Association of Cardio-Thoracic Surgery (EACTS) and the European Society of Cardiology (ESC), in collaboration with the European Association of Percutaneous Cardiovascular Interventions (EAPCI). Eur Heart J. 2008; 29 (11): 1463-70.

12. Perin MA, Brito FS Jr, Almeida BO, Pereira MA, Abizaid A, Tarasoutchi F, et al. Percutaneous aortic valve replacement for the treatment of aortic stenosis: early experience in Brazil. Arq Bras Cardiol. 2009; 93 (3): 299-306.

13. Carabello BA. Timing of valve replacement in aortic stenosis: moving closer to perfection. Circulation. 1997; 95 (9): 2241-3.

14. Carabello BA. Clinical practice: aortic stenosis. N Engl J Med. 2002; 346 (9): 677-82.

15. Hammermeister K, Sethi GK, Henderson WG, Grover FL, Oprian C, Rahimtoola $\mathrm{SH}$. Outcomes 15 years after valve replacement with a mechanical versus a bioprosthetic valve: final report of the Veterans Affairs randomized trial. J Am Coll Cardiol. 2000; 36 (4): 1152-8.

16. Amato MC, Moffa PJ, Werner KE, Ramires JA. Treatment decision in asymptomatic aortic valve stenosis: role of exercise testing. Heart. 2001;
86 (4): 381-6.

17. Monin JL, Lancellotti P, Monchi M, Lim P, Weiss E, Pierard L, et al. Risk score for predicting outcome in patients with asymptomatic aortic stenosis. Circulation. 2009; 120 (1): 69-75.

18. Otto CM, Burwash IG, Legget ME, Munt BI, Fujioka M, Healy NL, et al. Prospective study of asymptomatic valvular aortic stenosis. Clinical, echocardiographic, and exercise predictors of outcome. Circulation. 1997; 95 (9): 2262-70.

19. Pellikka PA, Nishimura RA, Bailey KR, Tajik AJ. The natural history of adults with asymptomatic, hemodynamically significant aortic stenosis. J Am Coll Cardiol. 1990; 15 (5): 1012-7.

20. Pellikka PA, Sarano ME, Nishimura RA, Malouf JF, Bailey KR, Scott CG, et al. Outcome of 622 adults with asymptomatic, hemodynamically significant aortic stenosis during prolonged follow-up. Circulation. 2005; 111 (24): 3290-5.

21. Rosenhek R, Binder T, Porenta G, Lang I, Christ G, Schemper M, et al. Predictors of outcome in severe, asymptomatic aortic stenosis. N Engl J Med. 2000; 343 (9): 611-7.

22. Bergler-Klein J, Klaar U, Heger M, Rosenhek R, Mundigler G, Gabriel H, et al. Natriuretic peptides predict symptom-free survival and postoperative outcome in severe aortic stenosis. Circulation. 2004; 109 (19): 2302-8.

23. Coglianese EE, Davidoff R. Predicting outcome in patients with asymptomatic aortic stenosis. Circulation. 2009; 120 (1): 9-11.

24. Amato MC, Moffa PJ. Prognosis of asymptomatic aortic valve stenosis evaluated with exercise test. Arq Bras Cardiol. 1998; 70 (4): 251-5.

25. Pierri H, Nussbacher A, Décourt LV, Medeiros C, Cattani A, Serro-Azul JB, et al. Clinical predictors of prognosis in severe aortic stenosis in unoperated patients $>$ or $=75$ years of age. Am J Cardiol. 2000; 86 (7): 801-4.

26. Pierri H, Décourt LV, Wajngarten M, Gebara O, Serro-Azul JB, Nussbacher A, et al. Perfil clínico de idosos portadores de estenose aórtica severa. Arq Bras Cardiol. 1997; 68 (6): 415-20.

27. Lancellotti P, Lebois F, Simon M, Tombeux C, Chauvel C, Pierard LA. Prognostic importance of quantitative exercise Doppler echocardiography in asymptomatic valvular aortic stenosis. Circulation. 2005; 112 (9 Suppl): 1377-1382.

28. Lung B, Baron G, Butchart EG, Delahaye F, Gohlke-Barwolf C, Levang OW, et al. A prospective survey of patients with valvular heart disease in Europe: The Euro Heart Survey on Valvular Heart Disease. Eur Heart J. 2003; 24 (13): $1231-43$

29. Rafique AM, Biner S, Ray I, Forrester JS, Tolstrup K, Siegel RJ. Meta-analysis of prognostic value of stress testing in patients with asymptomatic severe aortic stenosis. Am J Cardiol. 2009;104 (7): 972-7.

30. Dal-Bianco JP, Sengupta PP, Khandheria BK. Role of echocardiography in the diagnosis and management of asymptomatic severe aortic stenosis. Expert Rev Cardiovasc Ther. 2008; 6 (2): 223-33.

31. Dal-Bianco JP, Khandheria BK, Mookadam F, Gentile F, Sengupta PP. Management of asymptomatic severe aortic stenosis. J Am Coll Cardiol. 2008; 52 (16): 1279-92.

32. Carabello BA, Paulus WJ. Aortic stenosis. Lancet. 2009; 373 (9667): 956-66.

33. Lung B. Management of the elderly patient with aortic stenosis. Heart. 2008; 94 (4): 519-24 